\title{
The Effect of Inclusions on the Morphology of Fe-Ti Based Simulated Weld Metals
}

\author{
S. Talas, R.C. Cochrane*
}

Afyon Kocatepe University, Technical Education Faculty, Afyon, Turkey

*Department of Materials, University of Leeds, Leeds, UK

The effect of $\mathrm{Ti}$ in steel has been the centre of research due especially to its effect on the final microstructure, which is usually associated with acicular ferrite. For its formation, various mechanisms have been put forward by researchers [1-3] and, some concluded that its formation is related to the presence of inclusions [4] and they only act as passive nucleation sites to assist the heterogeneous nucleation of ferrite in undercooled welds. To date, some of the more favourable inclusions types are $\mathrm{TiO}$ and TiN [4] which are believed to influence the nucleation characteristics of ferrite in favour of epitaxial nucleation mechanism. Nevertheless, the effects of elements are not fully understood because of complex production methods. For example, the use of Si and Al to reduce O levels in steels may lead to confusion in understanding the effect of desired elements due to interaction between each element.

High purity Fe powder based alloys were re-melted using a modified TIG welding source in a closed Ar environment at constant cooling rate. Specimens were examined by SEM operated under $20 \mathrm{kV}$ fitted with Oxford Link EDS. By controlling the powder processing and melting time the $\mathrm{O}$ and $\mathrm{Ti}$ contents of the alloys could be varied independent of each other as shown in Table 1. No additions of $\mathrm{Al}$ or $\mathrm{Si}$ were made to ensure that only deoxidation reactions by $\mathrm{Ti}$ occurred. Duplication of an arbitrary submerged arc weld composition, FeCMnTi, gave microstructures indistinguishable from that of conventional weld; $\mathrm{C}$ and $\mathrm{Mn}$ additions also mimicked the known behaviour in welding. With increasing Ti additions to the Fe-Mn-C-Ti alloy, a range of microstructures from coarse to fine acicular ferrite was obtained as in the work by Evans [4]. The elimination of C and Mn, FeTi50, however, led to the formation of a structure analogous to that of acicular ferrite when sufficient Ti was added. SEM image in Figure 1 shows ferrite laths formed within the grain, which apparently shows intragranular formation of acicular laths, which may have been subjected to a recovery process on cooling. Further elimination of $\mathrm{O}$ caused the structural feature to disappear and it was replaced by an entirely polygonal ferrite microstructure (Figure 2). The inclusion type did not change markedly during this process but the number, density (and size) changed dramatically. In Figure 2, also fast moving solidification front led to the formation of some substructures which were probably affected by the presence of structural defects, such as inclusions. The implication of this study is that both inclusions and $\mathrm{Ti}$ in solution in austenite prior to transformation are essential prerequisites for the formation of acicular ferrite.

\section{References}

[1] Ricks, R.A., et al., J. Mats. Sci., 17 (1982) 732

[2] Shim Y., et al., Scripta Mater., 44 (2001) 49

[3] Zhang, Z. and Farrar, R.A., Mats. Sci. Tech. 12 (1996) 237

[4] G.M. Evans and N. Bailey, Metallurgy of Basic Weld Metal, Abington Publ., Cambridge, 1997 
Table 1. Compositions of specimens produced (in wt\%) (P:Purified)

\begin{tabular}{|c|c|c|c|c|}
\hline Code & $\mathrm{C}$ & $\mathrm{Mn}$ & $\mathrm{Ti}$ & $\mathrm{O}$ \\
\hline FeCMnTi & 0.06 & 1.4 & $0.02-0.084$ & $0.02-0.006$ \\
\hline FeTi50 & - & - & 0.085 & N/A \\
\hline FeTi50P & - & - & 0.09 & N/A \\
\hline
\end{tabular}

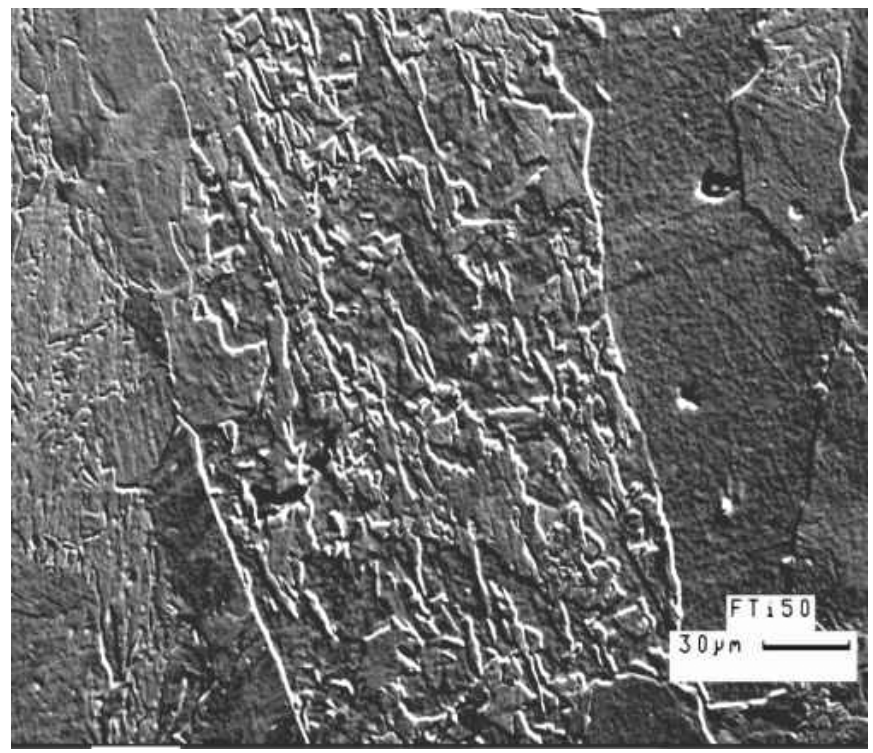

Figure 1. FeTi50 showing slightly recovered acicular ferrite laths inside a grain

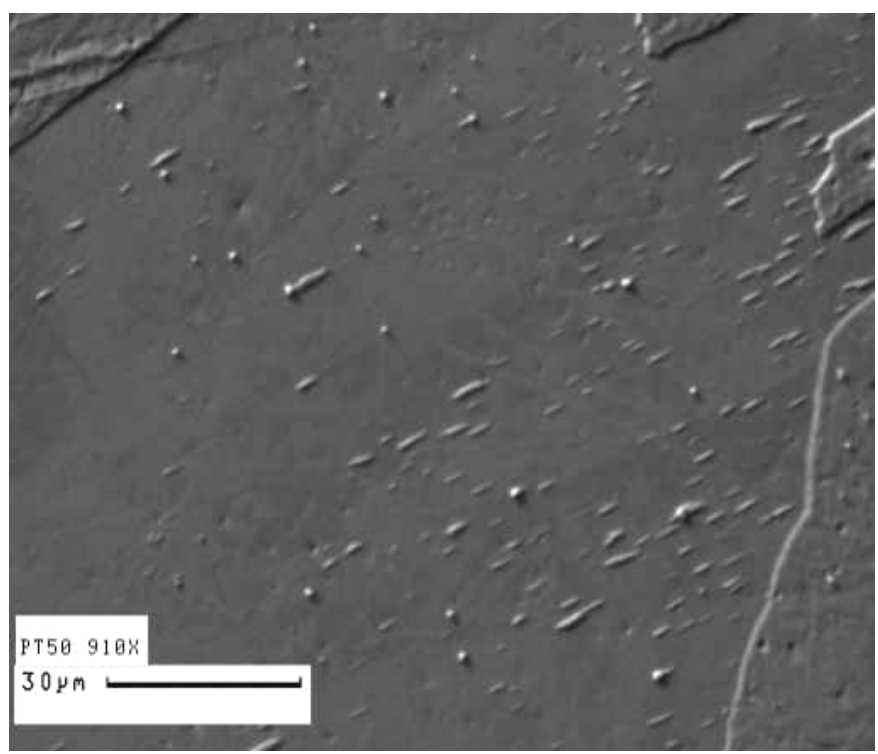

Figure 2. Purified FeTi50 SEM image showing substructures formed due probably to fast moving solidification front 